\title{
BMJ Open Scoping review protocol documenting cancer outcomes and inequalities for adults living with intellectual and/or developmental disabilities
}

\author{
Morgan Stirling (D) , Janice Linton, ${ }^{1}$ Hélène Ouellette-Kuntz, ${ }^{2}$ Shahin Shooshtari, ${ }^{1}$ \\ Julie Hallet, ${ }^{3}$ Christine Kelly, ${ }^{1}$ David Dawe, ${ }^{4,5}$ Mark Kristjanson, ${ }^{6,7}$ \\ Kathleen Decker, ${ }^{1,8}$ Alyson Mahar ${ }^{1}$
}

To cite: Stirling M, Linton J, Ouellette-Kuntz $\mathrm{H}$, et al. Scoping review protocol documenting cancer outcomes and inequalities for adults living with intellectual and/or developmental disabilities. BMJ Open 2019;9:e032772. doi:10.1136/ bmjopen-2019-032772

- Prepublication history and additional material for this paper are available online. To view these files, please visit the journal online (http://dx.doi. org/10.1136/bmjopen-2019032772).

Received 07 July 2019 Revised 20 September 2019 Accepted 15 October 2019

D Check for updates

(c) Author(s) (or their employer(s)) 2019. Re-use permitted under CC BY-NC. No commercial re-use. See rights and permissions. Published by BMJ.

For numbered affiliations see end of article.

\section{Correspondence to}

Dr Alyson Mahar;

alyson_mahar@cpe.umanitoba. ca

\section{ABSTRACT}

Introduction There is increasing attention on the cancer burden for adults with intellectual and developmental disabilities (IDD). Emerging evidence suggests there are differences in cancer experiences and outcomes for individuals living with IDD, from risk through survivorship. These differences may be attributed to features of the IDD, such as cognitive deficits and communication, as well as social determinants of health-like lower education levels and ableism. However, there is no comprehensive overview of the literature quantifying these potential disparities and describing the influencing factors. In this paper, we describe a scoping review protocol to systematically review published literature on cancer for adults with IDD. The purpose of this review is to identify differences in cancer risk, stage at diagnosis, treatment and survival along the cancer continuum for adults with IDD and outline potential contributing factors creating these disparities.

Methods and analysis We will follow Arksey and 0'Malley's expanded framework for scoping reviews to conduct this review. We will systematically search electronic databases for peer-reviewed, published journal articles to identify appropriate studies in collaboration with a health science librarian. Two reviewers will independently review titles and abstracts followed by a full-text review to determine whether it meets inclusion criteria. A data chart for collecting and sorting information will be developed in consultation with the team. Results will be collated and reported following the Preferred Reporting Items for Systematic Reviews and Meta-Analyses (PRISMA) extension for scoping reviews: PRISMA-Scoping Reviews. Extracted information will be summarised quantitatively and qualitatively to meet review objectives.

Ethics and dissemination This scoping review will employ a methodology to identify literature related to cancer outcomes and experiences for adults with IDD. Results will be disseminated to relevant stakeholders who care for and support individuals with IDD at local, provincial and national levels and through publishing findings. By highlighting the disparities in the cancer system and gaps in the research, this scoping review can provide direction for future action.
Strengths and limitations of this study

- This will be the first scoping review to systematically search outcomes for individuals with intellectual and developmental disabilities (IDD) along the cancer continuum from risk to survivorship/end-of-life.

- This review will map and describe the factors including social determinants of health that influence cancer outcomes and experiences of adults with IDD.

- As this is a scoping review, quality assessment and grading will not be performed, minimising the ability to provide clinical recommendations to improve cancer care for persons with IDD.

- Studies published in English and French will be included.

\section{INTRODUCTION}

There is greater attention and focus on the burden of cancer among individuals with intellectual and developmental disabilities (IDD). IDD, including Down syndrome, cerebral palsy and autism spectrum disorder, are life-long disorders originating before the age of 18 and affect $1 \%-3 \%$ of the population. IDD diagnoses range from mild to profound impairment with features such as diminished communication, difficulty processing information and problem solving, as well as challenges with activities of daily living. ${ }^{12}$ Significant improvements in life expectancy, ${ }^{34}$ the recognition that cancer is a leading cause of death in persons with IDD $^{56}$ and the possibility that significant limitations in intellectual functioning may make it challenging for persons with IDD to access and navigate a cancer setting ${ }^{127}$ are influencing this shift in focus.

Disparities in cancer outcomes persist within universal healthcare systems across the cancer control spectrum, from prevention to survivorship and end-of-life care. Many of the factors 
influencing these disparities are not connected to the cancer itself, but to a person's social and economic context that shape their ability to access cancer care. Groups most vulnerable to these disparities are ones that experience discrimination and underlying levels of economic, social and geographic disadvantage. ${ }^{8}$ Cancer risk factors, screening rates, stage at diagnosis, receipt of guidelinerecommended treatment, symptom management and cancer mortality vary by age, sex, immigration status, sexual orientation, socioeconomic position, geographic location, rurality and in indigenous populations. ${ }^{9-22}$ International, national and local cancer agencies have directed efforts to addressing barriers that lead to poorer outcomes and experiences for vulnerable populations. ${ }^{23-27}$ To make clinical care and delivery of support services more responsive to the needs of the vulnerable groups, there is an imperative to improve understanding of disparities within the cancer system. While this is an emerging area of research, there has been a lack of focus on the needs of adults with IDD, leaving many unanswered questions. ${ }^{28} 29$

Adults with IDD may be at greater risk of worse outcomes along the cancer continuum for a number of reasons. ${ }^{29} 30$ Potential pathways are related to features of IDD, that is, cognitive deficits and difficulties with communication and are influenced by the social determinants of health that shape one's economic and material reality. ${ }^{31}$ In particular, determinants such as provider bias and ableism; lower levels of employment, education and social support; lack of access to suitable transportation; a lack of provider knowledge and organisation of the health system that hinders access to services have been suggested as mechanisms leading to poorer outcomes. ${ }^{32-37}$ Existing literature reviews focusing on cancer and IDD to inform provider and system-level interventions are narrative in nature, ${ }^{28} 2938$ analyse a handful of studies on a particular cancer type e $^{38}$ or do not highlight modifiable barriers to improve cancer care, limiting actionable content. ${ }^{28} 2939$ This review seeks to address this gap and provide an overview of the literature on cancer outcomes among adults with IDD and the factors that influence them over the cancer continuum, from risk and prevention to end-of-life/survivorship.

The primary objectives of this scoping review are to systematically map and examine the evidence base comparing cancer outcomes for adults with IDD to those without, to explore the literature describing cancer care experiences for adults with IDD. It will focus primarily on cancer incidence, screening, staging, treatment and prognosis. Our goals are to direct future research efforts by identifying literature gaps and limitations, and highlight relevant social determinants of health influence cancer outcomes for individuals with IDD. It is our hope that this review will expand the wider body of research examining cancer disparities for vulnerable populations.

\section{METHODS AND ANALYSIS}

A scoping methodology approach will be used to explore the literature describing cancer outcomes for individuals with IDD. Scoping reviews can examine broad questions that aim to describe research activity in an area and its evidence base. ${ }^{40-42}$ We will follow the expanded Arksey and O'Malley (2005) framework for scoping reviews. ${ }^{40-42} \mathrm{We}$ will follow the Preferred Reporting Items for Systematic Reviews and Meta-Analyses (PRISMA) extension for reporting scoping reviews: PRISMA-Scoping Reviews checklist. $^{43}$

\section{Identifying the research question}

The scoping review will answer the questions:

1. What are the effects of living with an IDD on adult cancer risk, screening, stage at diagnosis, treatment and survival relative to those without IDD?

2. What factors related to features of IDD may contribute to differences in cancer care experiences and outcomes for individuals with IDD compared with those without?

3. What social determinants of health may contribute to differences in cancer care experiences and outcomes for individuals with IDD compared with those without?

The research team developed these questions and initial protocol in an iterative process and will revise the protocol as needed throughout the review process.

\section{Search strategy and information sources}

The primary search strategy was developed for Medline by the research team in collaboration with a health sciences librarian (JL). We will execute a similar search in Embase, Cochrane, CINAHL, Scopus and PsycINFO. The search will employ the following Medical Subject Headings (MeSH) headings for IDD and cancer, respectively, and will be linked with Boolean operator OR within a category and using the Boolean operator AND between IDD and cancer:

- IDD: [exp intellectual disability/], [exp Developmental Disabilities/]; [exp Child Development Disorders, Pervasive/].

- Cancer: $\exp [$ neoplasms/].

Preliminary searches in Medline and Embase identified 749 and 1916 potential citations, respectively. We have elected to complete a sensitive strategy given the lack of specificity in cancer-related MeSH headings for our research objectives. A copy of the search strategy has been included as a online supplementary file. We will restrict the study to those articles published in 2000 or later to best approximate the risk of cancer and cancer-related outcomes in adults living with IDD currently, given changes in IDD definitions and increased life expectancy over time. ${ }^{4}$ We will also review reference lists of relevant review articles and perform a cited reference search of included studies.

\section{Study selection}

Study selection will follow two stages. The first will be an initial review of titles and abstracts by two reviewers. The initial review will be done independently, with reviewers discussing results once screening is complete. Resolving disagreement will be attempted first by the two reviewers, 
Table 1 Inclusion and exclusion criteria to be applied to potentially eligible citations identified by the literature search

\section{Inclusion criteria}

Population Adults (age $\geq 18$ ) with an intellectual and/or developmental disability \pm a comparison group of adults with cancer and without an IDD.

Concept

Any cancer diagnosis occurring age $\geq 18$ Studies reporting cancer outcomes or experiences occurring along the continuum, including risk, screening, diagnosis, treatment, end-of-life/ survivorship and survival.

Studies where multiple health outcomes are reported, but cancer outcomes must be reported separately.

Biological, anatomical and/or social characteristics contributing to cancer outcomes or experiences for individuals with IDD.

$\begin{array}{ll}\text { Context } & \text { English and French } \\ & \text { Original research articles (Random Controlled Trials, } \\ & \text { cohort study, case-control and cross-sectional) } \\ & \text { Qualitative or quantitative } \\ & \text { Papers published after } 2000 .\end{array}$

IDD, intellectual and developmental disabilities.

but if necessary a third member of the research team will be consulted to reach consensus. Once the initial decision on which articles to include, we will begin the second stage of conducting a full-text review. Two reviewers will assess the articles to determine whether it meets the inclusion criteria. Those articles where there is uncertainty will be reviewed by a third member of the team to determine its eligibility. Table 1 outlines the inclusion/exclusion criteria following the population, concept and context categories for scoping reviews. ${ }^{44}$ Due to expertise within the team, we will include studies in both English and French.

\section{Charting the data}

A data chart will be developed in consultation with the research team. This chart will facilitate the collection and sorting of information from selected studies. Key areas that will be included in this chart will include: description of study population (eg, age, sex, socioeconomic status and comorbidity), definition of IDD, IDD diagnosis, context of IDD care (eg, institution and community), which milestone along the cancer continuum is explored in the study, cancers included (eg, lung, breast and melanoma), sources of information (eg, hospital records and cancer registry), study time frame, study location and sample size. For studies where the study population is not adults with IDD and is their caregiver or healthcare provider, this information will be noted. Cancer incidence, prevalence, screening rates, stage at diagnosis, treatment (eg, chemotherapy, radiation and surgery), survival from diagnosis or other relevant timepoints, palliation, follow-up care during

\section{Exclusion criteria}

Cancer outcomes for adults are not reported separately from children.

IDD definition is not consistent with current approaches or cannot be confirmed.

Studies on health of adults with IDD where no cancerrelated outcomes are reported/cancer-related outcomes not reported separately.

Primary focus is the relationship between cancer genes/ pathways and IDD. remission, recurrence rates and quality of life measures will be quantified and measures of comparison to the general cancer population extracted where possible. Major themes from qualitative studies will be extracted and summarised across studies. Where possible we will summarise quantitative and qualitative data within and across IDD diagnoses as well as within and across countries. The data chart will be piloted by two reviewers, with differences in charting to be resolved by a third member of the research team. After initial pilot, results will be shared with the research team to determine whether the data chart satisfactorily captures information to respond to research questions. Revisions will be incorporated if determined necessary.

\section{Collating, summarising and reporting the results}

Results will be summarised both quantitatively and qualitatively to provide a description of collected data. Descriptive statistics indicating frequencies and central measures of tendency will be used to report the number of studies reporting outcomes or care experiences at each stage of the cancer continuum, as well as the type of contributing characteristic(s) such as biological, social or economic factors will also be reported. The descriptive statistics will be reported in tables. A narrative synthesis of the findings will also be reported.

\section{Consultation}

We will engage with relevant stakeholders, including individuals within the IDD community such as individuals with IDD, their family members and service providers, as 
well as community organisations. to determine suitable approaches for dissemination and additional knowledge translation activities.

\section{Patient and public involvement}

Patients and the public were not involved in the development of this protocol.

\section{CONCLUSION}

This protocol outlines a rigorous method to systematically search and map the literature on cancer outcomes and care for individuals with IDD. To the best of the research team's knowledge, this is the first review that attempts to explore this area in any great detail. Significantly, this scoping review has the potential to highlight gaps and limitations in the existing body of research, and as such can provide direction for future inquiry.

\section{Author affiliations}

${ }^{1}$ Department of Community Health Sciences, University of Manitoba, Winnipeg, Manitoba, Canada

${ }^{2}$ Department of Public Health Sciences, Queen's University, Kingston, Ontario, Canada

${ }^{3}$ Department of Surgery, University of Toronto, Toronto, Ontario, Canada

${ }^{4}$ Internal Medicine, University of Manitoba College of Medicine, Winnipeg, Manitoba, Canada

${ }^{5}$ Hematology and Medical Oncology, CancerCare Manitoba, Winnipeg, Manitoba,

Canada

${ }^{6}$ Community Oncology Program, CancerCare Manitoba, Winnipeg, Manitoba, Canada ${ }^{7}$ Department of Family Medicine, University of Manitoba, Winnipeg, Manitoba, Canada

${ }^{8}$ Epidemiology, CancerCare Manitoba, Winnipeg, Manitoba, Canada

Contributors MS and AM conceived of and developed the protocol. JL, AM and MS conceived of the search strategy. MS, AM, HO-K and CK provided feedback to inform the protocol's theoretical foundation. AM executed the search. MS, JL, HO-K, $\mathrm{SS}, \mathrm{JH}, \mathrm{CK}, \mathrm{DD}, \mathrm{MK}, \mathrm{KD}$ and AM contributed to the development of the inclusion/ exclusion criteria and to the final manuscript.

Funding This project was funded through an unrestricted Research Start-Up Funds grant from the Office of the Vice President (Research \& International) at the University of Manitoba (Mahar).

Competing interests None declared.

Patient consent for publication Not required.

Provenance and peer review Not commissioned; externally peer reviewed.

Open access This is an open access article distributed in accordance with the Creative Commons Attribution Non Commercial (CC BY-NC 4.0) license, which permits others to distribute, remix, adapt, build upon this work non-commercially, and license their derivative works on different terms, provided the original work is properly cited, appropriate credit is given, any changes made indicated, and the use is non-commercial. See: http://creativecommons.org/licenses/by-nc/4.0/.

ORCID iD

Morgan Stirling http://orcid.org/0000-0002-4286-0688

\section{REFERENCES}

1 Eunice Kennedy Shriver National Institute of Child Health and Human Development. Intellectual and developmental Disabilites (IDDs): condition information: National Institutes of health, 2018. Available: https://www.nichd.nih.gov/health/topics/idds/conditioninfo/default [Accessed 27 Aug 2018].

2 Weiss R. Intellectual disability and developmental disorders in children. Introduction to abnormal child and adolescent psychology. USA: SAGE Publications Inc, 2018: 88-126.
3 Stankiewicz E, Ouellette-Kuntz H, Mclsaac M, et al. Patterns of mortality among adults with intellectual and developmental disabilities in Ontario. Can J Public Health 2018;109:866-72.

4 Coppus AM. People with intellectual disability: what do we know about adulthood and life expectancy? Dev Disabil Res Rev 2013;18:6-16.

5 Brameld K, Spilsbury K, Rosenwax L, et al. Use of health services in the last year of life and cause of death in people with intellectual disability: a retrospective matched cohort study. BMJ Open 2018;8:e020268.

6 Glover G, Williams R, Heslop P, et al. Mortality in people with intellectual disabilities in England. J Intellect Disabil Res 2017;61:62-74

7 Black DW. DSM-5® Guidebook the essential companion to the diagnostic and statistical manual of mental disorders. 5th edn American Psychiatric Publishing, 2014.

8 Kilbourne AM, Switzer G, Hyman K, et al. Advancing health disparities research within the health care system: a conceptual framework. Am J Public Health 2006;96:2113-21.

9 Bone A, McGrath-Lone L, Day S, et al. Inequalities in the care experiences of patients with cancer: analysis of data from the National cancer patient experience survey 2011-2012. BMJ Open 2014;4:e004567.

10 Clegg LX, Reichman ME, Miller BA, et al. Impact of socioeconomic status on cancer incidence and stage at diagnosis: selected findings from the surveillance, epidemiology, and end results: national longitudinal mortality study. Cancer Causes Control 2009;20:417-35.

11 Maddison AR, Asada Y, Urquhart R. Inequity in access to cancer care: a review of the Canadian literature. Cancer Causes Control 2011:22:359-66.

12 Ford JG, Howerton MW, Lai GY, et al. Barriers to recruiting underrepresented populations to cancer clinical trials: a systematic review. Cancer 2008;112:228-42.

13 Ward E, Jemal A, Cokkinides V, et al. Cancer disparities by race/ethnicity and socioeconomic status. CA Cancer J Clin 2004;54:78-93.

14 Decker KM, Kliewer EV, Demers AA, et al. Cancer incidence, mortality, and stage at diagnosis in first nations living in Manitoba. Current Oncology 2016;23:225-32.

15 Macleod U, Mitchell ED, Burgess C, et al. Risk factors for delayed presentation and referral of symptomatic cancer: evidence for common cancers. Br J Cancer 2009;101:S92-101.

16 Greenlee RT, Howe HL. County-level poverty and distant stage cancer in the United States. Cancer Causes Control 2009;20:989-1000.

17 Quinn GP, Sanchez JA, Sutton SK, et al. Cancer and lesbian, gay, bisexual, transgender/transsexual, and queer/questioning (LGBTQ) populations. CA Cancer J Clin 2015;65:384-400.

18 Davis LE, Bubis LD, Mahar AL, et al. Patient-reported symptoms after breast cancer diagnosis and treatment: a retrospective cohort study. Eur J Cancer 2018;101:1-11.

19 Mahar AL, Davis LE, Bubis LD, et al. Factors associated with receipt of symptom screening in the year after cancer diagnosis in a universal health care system: a retrospective cohort study. Curr Oncol 2019;26.

20 Bubis LD, Davis L, Mahar A, et al. Symptom burden in the first year after cancer diagnosis: an analysis of patient-reported outcomes. Journal of Clinical Oncology 2018;36:1103-11.

21 Sinding C, Warren R, Fitzpatrick-Lewis D, et al. Research in cancer care disparities in countries with universal healthcare: mapping the field and its conceptual contours. Support Care Cancer 2014;22:3101-20.

22 Weinstein LC, Stefancic A, Cunningham AT, et al. Cancer screening, prevention, and treatment in people with mental illness. CA Cancer $J$ Clin 2016;66:133-51.

23 Cancer CPA.. Canadian strategy for cancer control, 2019.

24 CancerCare Manitoba. 2016-2021 Manitoba cancer plan, 2016.

25 Cancer Care Ontario. Ontario cancer plan 5: 2019-2023, 2019.

26 de Souza JA, Hunt B, Asirwa FC, et al. Global health equity: cancer care outcome disparities in high-, middle-, and low-income countries. J Clin Oncol 2016;34:6-13.

27 WK S, Chan RJ, Truant T, et al. Global perspectives on cancer health disparities: impact, utility, and implications for cancer nursing. Asia Pac J Oncol Nurs 2016;3:316-23.

28 Hogg J, Tuffrey-Wijne I. Cancer and intellectual disability: a review of some key contextual issues. J Appl Res Intellect Disabil 2008;21:509-18.

29 Witham G, Haigh C. A narrative literature review examining cancer treatment issues for patients living with intellectual disabilities. Eur $J$ Oncol Nurs 2018;36:9-15. 
30 Robertson J, Hatton C, Baines S, et al. Systematic reviews of the health or health care of people with intellectual disabilities: a systematic review to identify gaps in the evidence base. J Appl Res Intellect Disabil 2015;28:455-523.

31 Mikkonen J, Raphael D. Social determinants of health: the Canadian facts. Toronto: York University School of Health Policy Management, 2010.

32 Ouellette-Kuntz H, Shooshtari S, Balogh R, et al. Understanding information about mortality among people with intellectual and developmental disabilities in Canada. J Appl Res Intellect Disabil 2015;28:423-35.

33 Meddings J, Reichert $\mathrm{H}$, Smith $\mathrm{SN}$, et al. The impact of disability and social determinants of health on Condition-Specific readmissions beyond Medicare risk adjustments: a cohort study. J Gen Intern Med 2017;32:71-80.

34 Emerson E, Madden R, Graham H, et al. The health of disabled people and the social determinants of health. Public Health 2011:125:145-7.

35 Krahn GL, Hammond L, Turner A. A cascade of disparities: health and health care access for people with intellectual disabilities. Ment Retard Dev Disabil Res Rev 2006;12:70-82.

36 Ouellette-Kuntz $\mathrm{H}$. Understanding health disparities and inequities faced by individuals with intellectual disabilities. J Appl Res Int Dis 2005;18:113-21.
37 Sullivan WF, Diepstra H, Heng J, et al. Primary care of adults with intellectual and developmental disabilities. Can Fam Physician 2018:64:254-79.

38 Willis DS, Kennedy Catriona M, Kilbride L, et al. Breast cancer surveillance in women with intellectual disabilities. Int J Disabil Hum Dev 2008;7:405-11.

39 Satgé D, Kempf E, Dubois J-B, et al. Challenges in diagnosis and treatment of lung cancer in people with intellectual disabilities: current state of knowledge. Lung Cancer Int 2016;2016:1-7.

40 Arksey H, O'Malley L. Scoping studies: towards a methodological framework. Int J Soc Res Methodol 2005;8:19-32.

41 Colquhoun HL, Levac D, O'Brien KK, et al. Scoping reviews: time for clarity in definition, methods, and reporting. $J$ Clin Epidemiol 2014;67:1291-4.

42 Levac D, Colquhoun H, O'Brien KK. Scoping studies: advancing the methodology. Implement Sci 2010;5.

43 Tricco AC, Lillie E, Zarin W, et al. PRISMA extension for scoping reviews (PRISMA-ScR): checklist and explanation. Ann Intern Med 2018;169:467-73.

44 Peters MDJ, Godfrey CM, Khalil H, Mclnerney K, et al. Guidance for conducting systematic scoping reviews. Int J Evid Based Healthc 2015;13:141-6. 